\title{
Interplay between grain boundary segregation and electrical resistivity in dilute nanocrystalline $\mathrm{Cu}$ alloys
}

\author{
Gyuseok Kim ${ }^{1}$, Xuzhao Chai ${ }^{2,3}$, Le Yu $^{2,3}$, Xuemei Cheng ${ }^{2}$, Daniel S. Gianola ${ }^{1,4^{*}}$ \\ ${ }^{1}$ Department of Materials Science and Engineering, University of Pennsylvania, Philadelphia, \\ PA, USA \\ ${ }^{2}$ Department of Physics, Bryn Mawr College, Bryn Mawr, PA, USA \\ ${ }^{3}$ School of Electronic Science and Engineering, Nanjing University, Nanjing, China \\ ${ }^{4}$ Materials Department, University of California, Santa Barbara, CA, USA \\ *Email: gianola@engr.ucsb.edu
}

\begin{abstract}
The relationships between microstructure, controlled by alloying elements prone to grain boundary segregation, and electrical resistivity in sputtered nanocrystalline $\mathrm{Cu}$ were investigated. We find a non-monotonic dependence of the mean grain size on solute concentration for both $\mathrm{Cu}-\mathrm{Nb}$ and $\mathrm{Cu}-\mathrm{Fe}$ dilute alloys, with a concentration regime where the grain size increases over that of pure $\mathrm{Cu}$ before refining with further alloying. The electrical resistivity follows the same trend, suggesting a non-equilibrium processing route that remarkably gives rise to dilute nanocrystalline $\mathrm{Cu}$ alloys with lower resistivity, thermal stability, and enhanced mechanical properties relative to their pure nanocrystalline counterpart.

Nanocrystalline (NC) metals have been the subject of intense research activity, driven largely by technological interests in their high hardness and strength. The results from decades of experiments and simulations point to the governing role of deformation physics unique to its coarse-grained counterparts, including grain boundary (GB) sliding, nucleation of dislocations from GBs and their subsequent isolated propagation, GB rotation, and stress-assisted grain growth [1,2]. Owing to the large volume fraction of material in near-GB regions in nanocrystalline metals, the properties of these materials are governed by interfacial phenomena.
\end{abstract}


In parallel with new insights on deformation mechanisms, the technological use of metallic thin films and coatings as electrical interconnects and structural features in MEMS/NEMS, which often are nanostructured by virtue of the non-equilibrium processing routes used to synthesize them, necessitates a proper optimization of both electrical and mechanical properties. For instance, interconnect materials with dimensions that are ever-miniaturizing require low electrical resistivity so as to cope with thermal management from Joule heating at ultra-high current densities [3]. Furthermore, reliability concerns focus on mitigation of electromigration, thermal stress and shock, and fatigue [4].

Despite an emerging understanding of deformation physics and concomitant properties, the majority of studies have focused on nominally pure systems. The lack of understanding of mechanical and electrical behavior in more chemically-complex nanocrystalline metals largely limits the wide use of alloy systems. However, alloying is a practical reality; thus the complex interplay between length scale, interfacial, and alloying effects must be thoroughly understood. The current understanding of alloying effects are mostly focused on spatial distribution of solutes [5-9] with the aim of endowing nanocrystalline materials with thermal stability; these results show that the grain size and solutes are typically inversely correlated [10,11].

For applications where Ohmic losses are to be minimized, the role of solutes is largely a deleterious one, with the reduction in grain size and increased alloying content leading to interface and impurity scattering, respectively [12-15]. Correspondingly, thermal annealing of pure $\mathrm{Cu}$ is generally employed to reduce electrical resistivity during processing [13]. Thus, materials engineers are often faced with a compromise between thermal stability, electrical conductivity, mechanical reliability, and the feasibility of high temperature processing in cases where flexible polymeric substrates are of interest.

In this study, we report on the use of co-sputtering of pure $\mathrm{Cu}$ and with $\mathrm{Cu}-\mathrm{M}(\mathrm{M}=\mathrm{Nb}$ and $\mathrm{Fe})$ alloys to produce non-monotonic grain refinement in nanocrystalline copper alloys. Detailed microstructural characterization suggests that the apparent breakdown of the inverse correlation between grain size and alloying content in the dilute regime is caused by the competition between the internal driving force from solute misfit strain and solute drag effects. These nonequilibrium effects enabled by sputtering deposition methods are linked to the spatial distribution 
of solute atoms, adding another dimension to microstructural and chemical control in nanocrystalline alloys. We apply this unique microstructural and chemical control to tailor the electrical conductivity of $\mathrm{Cu}$ thin films for interconnect applications, where deleterious increases in resistivity with decreasing device size (leading to lowered energy efficiencies and increased parasitic heating) pose a substantial challenge. These insights are used to produce thermally stable nanocrystalline alloy thin films with both lower electrical resistivity and higher hardness than their pure nanocrystalline $\mathrm{Cu}$ counterpart.

NC pure and alloyed copper thin films were deposited onto high purity Si (001) dies with nominal widths of $20 \mathrm{~mm}$ using physical vapor deposition (AJA ATC Orion Sputtering Deposition System) to co-sputter from pure $\mathrm{Cu}$ and $\mathrm{Cu}_{96} \mathrm{M}_{4}$ alloy targets, where $\mathrm{M}$ is either $\mathrm{Nb}$ or Fe. The targeted compositional range of solutes was 0 to 4 at.\%. To minimize unwanted impurity contamination, we used high purity sputtering targets $(99.999 \%$ for $\mathrm{Cu}, 99.95 \%$ for $\mathrm{CuNb}$ and $\mathrm{CuFe}$ ) and only performed synthesis when the chamber base pressure reached $<7 \times 10^{-8}$ mbar. Sputtering deposition was performed at an Ar pressure of $5.2 \times 10^{-3} \mathrm{mbar}$. The sample stage was continuously rotated during sputtering to achieve uniformity of the thin films. To control the composition of the sputtered binary alloy films, the power applied to each sputtering target was adjusted as listed in Table 1. The growth rate of the thin films was maintained between 0.10 and $0.15 \mathrm{~nm} / \mathrm{s}$. Proper sputtering time was selected to control the film thickness to be nominally between 50 and $60 \mathrm{~nm}$. To confirm the reproducibility of the measured properties of the thin films, 35 batches of samples were deposited. Energy dispersive spectroscopy (EDS) and focused ion beam (FIB, FEI Strata DB 235) were used to measure the composition and the thickness of films, respectively. The microstructure of the films was further investigated with transmission electron microscopy (TEM, JEOL 2100). Quantitative grain size analyses for both alloy systems were performed with plan-view dark field TEM images. At least 600 grains were measured for each composition, from which full grain size statistics were calculated. Ratios of the lattice constants of the $\mathrm{Cu}$ alloys to pure $\mathrm{Cu}$ were obtained by measuring the relative diameter of the diffraction rings visible in each thin film and performing linear regression of the relative diameter to the composition.

A 4-point electrical probe method with a truncated conical tip outfitted with $50 \mu \mathrm{m}$ radius of sphere was employed to measure the average sheet resistance of the thin films (Cascade 4 point 
probe, Keithley 2000 multi-meter, Agilent E3612A power supply). This measurement technique provided an accurate resistance value by eliminating the error associated with the internal and contact resistances stemming from the probe tips and leads. The obtained sheet resistances were corrected by multiplying the values by the sample size correlation factor [16], and accounting for the average thickness of each thin film as measured by cross sectioning with the FIB and subsequent SEM imaging.

The representative sputtering conditions for each batch and the resultant thickness, composition and grain size are listed in Table 1. Alloying contents as high as 3.2 at.\% and 2.6 at.\% were achieved for $\mathrm{Nb}$ and $\mathrm{Fe}$, respectively. In this range, the microstructure is truly nanocrystalline (Figure 1) with mean grain sizes in all films (both pure $\mathrm{Cu}$ and alloyed) falling well below $20 \mathrm{~nm}$.

The trends in grain size as a function of composition become quantitatively apparent when examined as cumulative distribution functions of grain sizes, as shown for $\mathrm{Cu}-\mathrm{Nb}$ (Figure 2(a)) and $\mathrm{Cu}-\mathrm{Fe}$ (Figure 2(b)), as well as mean grain sizes (Figure 2(c)). Grain coarsening occurs in the range of 0 to 1.5 at.\% $\mathrm{Nb}$ and 0 to 2.5 at.\% $\mathrm{Fe}$ (red shaded region). Specifically, whereas the mean grain size of the pure $\mathrm{Cu}$ film is $10.1 \mathrm{~nm}$, those of 1.14 at.\% $\mathrm{Nb}$ and 0.73 at.\% Fe increased to $13.6 \mathrm{~nm}$ (35\% increase) and $14.5 \mathrm{~nm}$ (44\% increase), respectively. Notably, we did not observe grain growth over time at room temperature, which implies that such a significant increase in grain size occurred as a consequence of the non-equilibrium nature of sputter deposition, without the need for subsequent thermal annealing or room temperature aging. This suggests that the spatial distribution of the solutes could be also a function of the global alloying content.

To understand the origin of the grain coarsening in the dilute regime for both alloys, the lattice parameters of $\mathrm{Cu}-\mathrm{Nb}$ films relative to those of pure $\mathrm{Cu}$ were measured from TEM diffraction patterns (Figure 2(d)). Lattice parameters were measured at two time intervals (3 and 90 days after deposition) to determine the influence of room temperature aging processes. Whereas the relative lattice parameters of the $\mathrm{Cu}-\mathrm{Nb}$ alloys measured shortly after 3 days are larger than that of pure $\mathrm{Cu}$ signifying a misfit strain from substitutional point defects, those measured 90 days after deposition were almost the same as that of pure $\mathrm{Cu}$ irrespective of the global $\mathrm{Nb}$ content. Since the diffraction patterns reflect the intragranular regions of the material where coherent 
scattering of electrons occurs and not those regions in close proximity to grain boundaries $[17,18]$, this result indicates that the solutes are more uniformly distributed in the material shortly after the sputtering, and over time subsequently segregate to grain boundaries (or potentially surfaces). This suggests that room temperature diffusion is relatively sluggish despite the strong tendency for grain boundary segregation (positive enthalpy of segregation) in both alloys. This can be reconciled by considering the low diffusivity of $\mathrm{Nb}$ in $\mathrm{Cu}$ at room temperature [19] which in our scenario could be somewhat accelerated given the expectation of a relatively large concentration of vacancies due to the non-equilibrium sputtering conditions and short diffusion lengths attributed to the very small grain sizes. Thereby, these mechanisms may help solutes redistribute from grain interiors to grain boundary regions over time scales of days, ultimately relaxing the grain interior misfit strains while modifying the grain boundary chemistry. The solutes residing in the grain interior generate a misfit strain magnitude that depends on the atomic mismatch between solvent and solute as described by Vegard's law, and quantified by a lattice misfit strain parameter, $\eta=(1 / a)(\delta a / \delta c)$, where $a$ is the lattice constant of the pure solvent and $c$ is the composition. Özerinç et al. reported values of $\eta=0.28$ in the $\mathrm{Cu}-\mathrm{Nb}$ alloy system cosputtered by PVD as obtained from X-ray diffraction experiments [20]. Our relative lattice parameter measured 3 days after deposition in Figure 2(d) gives $\eta=0.35$, which is in good agreement with the previous study [20]. This indicates that a certain concentration of solutes may reside in intragranular sites directly after sputtering, rather than rapidly diffusing or segregating in the grain boundary, causing the misfit strain in grain interior.

It is known that energetically unfavorable intragranular solutes with, for instance, large atomic mismatch with the solvent or positive enthalpy of segregation prefer to reside at grain boundaries since segregation of solutes can reduce the grain boundary energy [21,9,22]. The $\mathrm{Nb}$ and $\mathrm{Fe}$ solutes are immiscible in $\mathrm{Cu}$ at room temperature [23,24], with a positive enthalpy of segregation of $\mathrm{Nb}$ and $\mathrm{Fe}$ in $\mathrm{Cu}$ [25]. Thus, it is reasonable to presume that $\mathrm{Nb}$ and $\mathrm{Fe}$ solutes reside in the near vicinity of grain boundaries. The sputtering process, however, also produces nonequilibrium vacancy concentrations, and thereby intragranular sites for solutes to be located [26]. Moreover, the sputtering power for the alloy target is two to ten times smaller than that for pure copper. The significantly lower energy of the adsorbing alloying elements will consequently provide limited momentum that facilitates surface mobility enabling the solutes to segregate to 
grain boundaries [27,28]. As a result, the kinetically trapped intragranular solutes will lead to the generation of a misfit strain energy. This, in turn, results in the driving force for grain growth to relieve the stored excess energy [29]. Therefore, increasing the global content of solute will provide a greater driving force unless grain boundary segregation can occur. We note that grain growth caused by elastic anisotropy has a directional bias for grain boundary motion. In contrast, the grain growth mechanism suggested by our results is governed by a driving force arising from the heterogeneity of the strain field in the solid solution (analogous to recrystallization). On the other hand, the solutes retard the grain boundary migration necessary for grain growth by kinetic drag. The drag force $P$ caused by impurities can be expressed as $P=v k_{B} T / D$, where $v$ is the velocity of grain boundary, $k_{B}$ is the Boltzmann constant, $T$ is the temperature, $\Gamma$ is the number of excess impurities per unit area of grain boundary, and $D$ is the bulk diffusivity [30]. As the number of solutes increase or diffusivity decreases, the drag force increases. Taken as a whole, the competition between the roles of the increasing driving force for grain growth and the increasing drag force with increasing global alloying content presumably governs regimes of both grain growth and refinement.

In light of the competing mechanisms of misfit strain energy and kinetic solute drag, we propose a schematic view of the maximum grain size in our sputtered films as a function of composition (Figure 3). The grain size scales with the misfit strain energy, with a strength that depends on the atomic radius mismatch between solute and solvent. As our measured lattice misfit strain parameter of $\mathrm{Nb}$ in $\mathrm{Cu}$ is $\eta=0.35$ (similar to $\eta=0.28$ as reported in Ref. [25]) while that of Fe in $\mathrm{Cu}$ is $\eta=0.02$ [31], $\mathrm{Nb}$ solutes provide the greater driving force for grain growth per unit global content of solute. On the other hand, the grain size should scale inversely to kinetic solute drag. Since the diffusivity of $\mathrm{Nb}$ in $\mathrm{Cu}$ is lower than that of $\mathrm{Fe}$ in $\mathrm{Cu}[19,32], \mathrm{Nb}$ solutes will more strongly retard the migration of grain boundary. The net result of these competing mechanisms sets the non-monotonic shape of our measured grain sizes as a function of both $\mathrm{Nb}$ and Fe solute concentrations, consistent with our experimental results in Figure 2(c). Most importantly, our experimental results show that the mean grain size peaks in the dilute regime $(<1$ at. \%).

We next draw relationships between the microstructure and chemical variations and electrical properties of our sputtered films, which would be expected to be governed by both grain boundary and impurity scattering mechanisms. Measurements of the normalized electrical 
resistivity of our pure $\mathrm{Cu}$ and $\mathrm{Cu}$ alloy thin films are shown in Figure 4. Beginning with the pure copper films, we measured a resistivity value of $9.3 \mu \Omega-\mathrm{cm}$, similar to values reported in nanocrystalline $\mathrm{Cu}$ of approximately $7 \mu \Omega$-cm in films prepared via PVD [15] and $18 \mu \Omega$-cm in coatings prepared with electroplating methods [33]. The high resistivity in thin film or nanocrystalline form relative to that of bulk $(1.7 \mu \Omega-\mathrm{cm})$ is attributed to size effects associated with reduced thickness or grain sizes, as well as the potential influence of roughness $[12,13,15]$. We note that the resistivities of $\mathrm{Nb}$ samples are higher than $\mathrm{Cu}$ counterparts when synthesized using the same method $[34,35]$. Despite a report from Mahalingam et al. showing a resistivity drop in radio frequency sputtered $\mathrm{Cu}$ thin film with 2.7 at. $\% \mathrm{Nb}$, which was attributed to the low quality of the nominally pure $\mathrm{Cu}$ films (with an as-deposited resistivity of $\sim 35 \mu \Omega-\mathrm{cm}$ ) [36], the addition of $\mathrm{Nb}$ solutes in $\mathrm{Cu}$ generally leads to increases of resistivity as $\mathrm{Nb}$ provides additional scattering sites for electrons $[35,37,38]$.

In our study, we find that the resistivity non-monotonically varies with the content of solutes irrespective of the solute species, as shown in Figure 4. Strikingly, we find that, in the dilute regime ( 1.5 at $\% \mathrm{Nb}$ and $\mathrm{Fe})$, the resistivity drops by up to $36 \%$ with $\mathrm{Nb}$ solutes and $51 \%$ with $\mathrm{Fe}$ solutes relative to pure $\mathrm{NC} \mathrm{Cu}$ (red shaded region of Figure 4). Such behavior in resistivity can be primarily attributed to the microstructural changes previously described, most notably the increase in grain size. To understand these results, we estimate the resistivity changes predicted by the Fuchs-Sondheimer (FS) and Mayadas-Shatzkes (MS) phenomenological models which give the influence of thickness and grain size, respectively. Since the film thickness was kept fixed at $\sim 50 \mathrm{~nm}$, the contributions of the resistivity from thickness should be invariant to alloying content. We observe that the resistivity-grain size relationship in the dilute regime $(<1.5$ at.\%) approximately follows the trend predicted by a cumulative FS-MS model, suggesting that other effects such as scattering from solute atoms or vacancies [39] play a negligible role. At $\sim 1.3$ at.\% of solute, the resistivity of the alloy matches that of the pure $\mathrm{Cu}$ films. Beyond 1.5 at.\%, the resistivity monotonically increases, with the FS-MS model underpredicting both the absolute value of resistivity and its dependence on grain size, indicating that point defect scattering becomes substantial in addition to the grain refinement [39,47]. We hypothesize such discrepancies reflect the distinct spatial distribution of solutes (intra- vs. intergranular) which additionally mediate the resistivity [39]. This is further corroborated by the result that the 
resistivity uniformly decreases after 70 days of aging at room temperature, with a stronger reduction at higher solute concentrations. This suggests that over time, solutes diffuse toward GBs (consistent with lattice parameter measurements) thereby reducing intragranular point defect scattering sites. We note that the peaks of grain size and conductivity (minimum of resistivity) for the two alloying species are offset by $\sim 1$ at.\%, which may be explained by annihilation of vacancy by solutes [40]. The small amount of solute in the grain interior will lead to not only grain coarsening but also annihilation of vacancies. Details of the modeling and aging experiments will be discussed in a forthcoming publication. The striking implication of our results is that a concentration regime exists where the resistivity of $\mathrm{Cu}-\mathrm{Nb}$ and $\mathrm{Cu}-\mathrm{Fe}$ alloys is substantially lower than that of their pure $\mathrm{Cu}$ counterpart.

In summary, we investigated non-monotonic grain refinement in thin film $\mathrm{Cu}$ alloys with $\mathrm{Nb}$ and Fe solutes. The grain coarsening in the dilute alloy regime can be attributed to the competing effects of the driving force for grain growth from internal misfit strain and the pinning pressure from kinetic drag effects. The electrical resistivity of thin film $\mathrm{Cu}$ alloys decreased by as much as $36 \%$ and $51 \%$, relative to pure $\mathrm{Cu}$, with the addition of $\mathrm{Nb}$ and $\mathrm{Fe}$ solutes, respectively in dilute concentration regimes $(<1.5$ at $\%)$. The tailoring of electrical resistivity and grain size by adding solutes at room temperature provides a materials-based perspective on various applications where electrical [42], mechanical [20], and thermal [41] considerations predominate such as electronic devices, interconnects, and coating technologies [4]. Our method could produce mechanically robust and reliable [42] interconnect materials at room temperature, without the need for annealing steps that are not amenable to flexible electronic applications (e.g. wearable device and touch sensors atop polymer substrates $[43,44]$ ), and also paired with electrical resistivities not compromised by alloying.

\section{Acknowledgements}

This research was supported by the U.S. Department of Energy, Office of Basic Energy Sciences, Division of Materials Science and Engineering under Award \#DE-SC0008135. X. Cheng thanks partial support of the National Science Foundation under Award \# MRI, DMR-1126656 (thin film deposition). We thank the support of the staff and facilities at the Penn Nanoscale 
Characterization Facility and the Quattrone Nanofabrication Facility, both at the University of Pennsylvania. The authors also thank X. Wang for the assistance in sputtering. 


\section{Table}

Table 1. Representative sputtering conditions and resultant thickness, composition and grain size of films.

\begin{tabular}{|c|c|c|c|c|c|}
\hline \multirow[t]{2}{*}{ Solute } & \multicolumn{2}{|c|}{ Sputtering Power } & \multirow[t]{2}{*}{ Thickness (nm) } & \multirow{2}{*}{$\begin{array}{c}\text { Solute } \\
\text { Concentration } \\
(\text { at } \%)\end{array}$} & \multirow{2}{*}{$\begin{array}{c}\text { Mean Grain } \\
\text { size }(\mathrm{nm})\end{array}$} \\
\hline & $\mathrm{Cu}(\mathrm{W})$ & $\mathrm{Cu}_{96} \mathrm{M}_{4}(\mathrm{~W})$ & & & \\
\hline- & 200 & 0 & $58.2( \pm 1.1)$ & 0 & $10.1( \pm 5.9)$ \\
\hline \multirow[t]{4}{*}{$\mathrm{M}=\mathrm{Nb}$} & 200 & 50 & $46.4( \pm 1.8)$ & $0.64( \pm 0.08)$ & $12.2( \pm 7.0)$ \\
\hline & 200 & 100 & $46.8( \pm 2.3)$ & $1.14( \pm 0.13)$ & $13.6( \pm 7.9)$ \\
\hline & 150 & 200 & $47.7( \pm 1.9)$ & $1.96( \pm 0.06)$ & $7.9( \pm 4.3)$ \\
\hline & 0 & 200 & $60.0( \pm 1.9)$ & $3.20( \pm 0.10)$ & $7.8( \pm 4.9)$ \\
\hline \multirow[t]{5}{*}{$\mathrm{M}=\mathrm{Fe}$} & 200 & 20 & $69.4( \pm 2.2)$ & $0.46( \pm 0.16)$ & $13.2( \pm 7.1)$ \\
\hline & 200 & 50 & $61.0( \pm 3.0)$ & $0.73( \pm 0.24)$ & $14.5( \pm 8.6)$ \\
\hline & 200 & 100 & $63.2( \pm 1.6)$ & $1.08( \pm 0.37)$ & $12.5( \pm 6.1)$ \\
\hline & 150 & 200 & $57.8( \pm 3.6)$ & $1.41( \pm 0.46)$ & $12.3( \pm 6.0)$ \\
\hline & 0 & 200 & $53.0( \pm 3.0)$ & $2.58( \pm 0.07)$ & $10.0( \pm 4.5)$ \\
\hline
\end{tabular}




\section{Figure Captions}

Figure 1: Bring field -TEM images showing the microstructure of pure $\mathrm{Cu}$ and $\mathrm{Cu}$ alloys. (a) Pure $\mathrm{Cu}$ (b) $\mathrm{Cu}-0.64$ at.\% $\mathrm{Nb}$ (c) $\mathrm{Cu}-3.20$ at.\% Nb (d) $\mathrm{Cu}-0.73$ at.\% Fe (e) $\mathrm{Cu}-2.58$ at.\% Fe . The micrographs show that the grain sizes of dilute alloy samples are larger than those of pure $\mathrm{Cu}$. The insets are the corresponding diffraction patterns. The bright and discontinuous spots in diffraction ring in (b) and (d) also indicate the larger mean grain sizes of dilute alloy samples. The scale bar indicates $50 \mathrm{~nm}$.

Figure 2: Grain size statistics shown as cumulative area fraction of representative batches in the (a) $\mathrm{Cu}-\mathrm{Nb}$ system (b) Cu-Fe system. (c) Dependence of mean grain size on the solute concentration. The shaded areas indicate regimes of grain coarsening (red) and grain refinement (blue). Dashed lines are guides for the eye. (d) Change in lattice parameter of $\mathrm{CuNb}$ relative to pure $\mathrm{Cu}$ after 3 days and 90 days from sputtering deposition.

Figure 3: Schematic of maximum grain size $d_{\max }$ as a function of solute concentration. Two different mechanisms, internal misfit strain energy and kinetic solute drag, compete with each other to determine the resultant grain size.

Figure 4: Electrical resistivity of pure $\mathrm{Cu}$ and $\mathrm{Cu}$ alloy as a function of solute concentration. The shaded regions show where the resistivity is lower (red) or higher (blue) than that of the pure Cu films. The error bars represent standard deviations of resistivity (vertical) and EDS (horizontal) measurements. For comparison, the resistivities in $\mathrm{NC} \mathrm{Cu}$ from various references and bulk $\mathrm{Cu}$ resistivity are also plotted $[15,45,46]$. 


\section{References}

[1] M. a. Meyers, a. Mishra, D.J. Benson, Prog. Mater. Sci. 51 (2006) 427-556.

[2] T.J. Rupert, D.S. Gianola, Y. Gan, K.J. Hemker, Science 326 (2009) 1686-90.

[3] T. Gupta, Copper Interconnect Technology, Springer New York, New York, NY, 2009.

[4] R.J. Gutmann, T.P. Chow, A.E. Kaloyeros, W. a. Lanford, S.P. Muraka, Thin Solid Films 262 (1995) 177-186.

[5] M. He, P. Felfer, S. Dasgupta, S. Samudrala, P.J. Malone, Acta Mater. (2014).

[6] F. Tang, D.S. Gianola, M.P. Moody, K.J. Hemker, J.M. Cairney, Acta Mater. 60 (2012) 10381047.

[7] D.S. Gianola, S. Van Petegem, M. Legros, S. Brandstetter, H. Van Swygenhoven, K.J. Hemker, Acta Mater. 54 (2006) 2253-2263.

[8] T.J. Rupert, J.C. Trenkle, C.A. Schuh, Acta Mater. 59 (2011) 1619-1631.

[9] T. Chookajorn, H.A. Murdoch, C.A. Schuh, Science 337 (2012) 951-4.

[10] A.J. Detor, C.A. Schuh, J. Mater. Res. 22 (2007) 3233-3248.

[11] S.C. Mehta, D.A. Smith, U. Erb, Mater. Sci. Eng. A 204 (1995) 227-232.

[12] W. Wu, S.H. Brongersma, M. Van Hove, K. Maex, 2838 (n.d.) 10-13.

[13] T. Sun, B. Yao, A.P. Warren, K. Barmak, M.F. Toney, R.E. Peale, K.R. Coffey, Phys. Rev. B Condens. Matter Mater. Phys. 81 (2010) 1-12.

[14] W.L. Prater, E.L. Allen, W.-Y. Lee, M.F. Toney, J. Daniels, J. a. Hedstrom, Appl. Phys. Lett. 84 (2004) 2518.

[15] E. V. Barnat, D. Nagakura, P.I. Wang, T.M. Lu, J. Appl. Phys. 91 (2002) 1667-1672.

[16] F.M. Smits, Bell Syst. Tech. J. 37 (1958) 711-718.

[17] J. Markmann, V. Yamakov, J. Weissmueller, Scr. Mater. 59 (2008) 15-18.

[18] a. Stukowski, J. Markmann, J. Weissmüller, K. Albe, Acta Mater. 57 (2009) 1648-1654.

[19] D.. Bulrymowicz, J.R. Manning, M.E. Read, J. Phys. Chem. Ref. Data 4 (1975) 177.

[20] S. Özerinç, K. Tai, N.Q. Vo, P. Bellon, R.S. Averback, W.P. King, Scr. Mater. 67 (2012) 720-723.

[21] J. Weissmüller, Nanostructured Mater. 3 (1993) 261-272.

[22] F. Liu, R. Kirchheim, J. Cryst. Growth 264 (2004) 385-391.

[23] D.J. Chakrabarti, D.E. Laughlin, Bull. Alloy Phase Diagrams 2 (1982) 455-460.

[24] C.P. Wang, X.J. Liu, I. Ohnuma, R. Kainuma, K. Ishida, J. Phase Equilibria Diffus. 25 (2004) 320-328.

[25] Y. Ashkenazy, N.Q. Vo, D. Schwen, R.S. Averback, P. Bellon, Acta Mater. 60 (2012) 984-993.

[26] K.S. Sree Harsha, Principles of Vapor Deposition of Thin Films, 2006.

[27] M.-T. Le, Y.-U. Sohn, J.-W. Lim, G.-S. Choi, Mater. Trans. 51 (2010) 116-120. 
[28] K.-Y. Chan, B.-S. Teo, Microelectronics J. 37 (2006) 1064-1071.

[29] S. Edition, Grain Boundary Migration in Metals, 2010.

[30] J.W. Cahn, Acta Metall. 10 (1962) 789-798.

[31] E. Ma, M. Atzmon, F.E. Pinkerton, J. Appl. Phys. 74 (1993) 955.

[32] M.E.R. Daniel B. Butrymowicz, John R. Manning, J. Phys. Chem. Ref. Data 5 (1976) 103.

[33] L. Lu, Y. Shen, X. Chen, L. Qian, K. Lu, Science 304 (2004) 422-6.

[34] M. Fenn, G. Akuetey, P.E. Donovan, J. Physics-Condensed Matter 10 (1998) 1707-1720.

[35] R. Banerjee, S. Bose, A. Genc, P. Ayyub, J. Appl. Phys. 103 (2008).

[36] T. Mahalingam, C.H. Lin, L.T. Wang, J.P. Chu, Mater. Chem. Phys. 100 (2006) 490-495.

[37] K. Barmak, C. Cabral, K.P. Rodbell, J.M.E. Harper, J. Vac. Sci. Technol. B Microelectron. Nanom. Struct. 24 (2006) 2485.

[38] A. Gungor, K. Barmak, A.D. Rollett, C. Cabral, J.M.E. Harper, J. Vac. Sci. Technol. B Microelectron. Nanom. Struct. 20 (2002) 2314.

[39] C.Y. Ho, M.W. Ackerman, K.Y. Wu, T.N. Havill, R.H. Bogaard, R. a. Matula, S.G. Oh, H.M. James, J. Phys. Chem. Ref. Data 12 (1983) 183.

[40] J.Y. Kim, M.-W. Oh, S. Lee, Y.C. Cho, J.-H. Yoon, G.W. Lee, C.-R. Cho, C.H. Park, S.-Y. Jeong, Sci. Rep. 4 (2014) 5450.

[41] Y. Zhao, T.A. Furnish, M.E. Kassner, A.M. Hodge, J. Mater. Res. 27 (2012) 3049-3057.

[42] J.P. Gambino, 2010 17th IEEE Int. Symp. Phys. Fail. Anal. Integr. Circuits (2010) 1-7.

[43] J. van den Brand, M. de Kok, M. Koetse, M. Cauwe, R. Verplancke, F. Bossuyt, M. Jablonski, J. Vanfleteren, Solid. State. Electron. 113 (2015) 116-120.

[44] Y.-H. Shin, C.-K. Cho, H.-K. Kim, Thin Solid Films 548 (2013) 641-645.

[45] A.A.M. and F.R. de B. Y.K. Huang, Nanostructured Mater. 2 (1993) 505-513.

[46] G. Ramaswamy, A.K. Raychaudhuri, J. Goswami, S. a Shivashankar, J. Phys. D. Appl. Phys. 30 (1997) L5-L9.

[47] F.T.N. Vüllers, R. Spolenak, Acta Mater. 99 (2015) 213-227. 

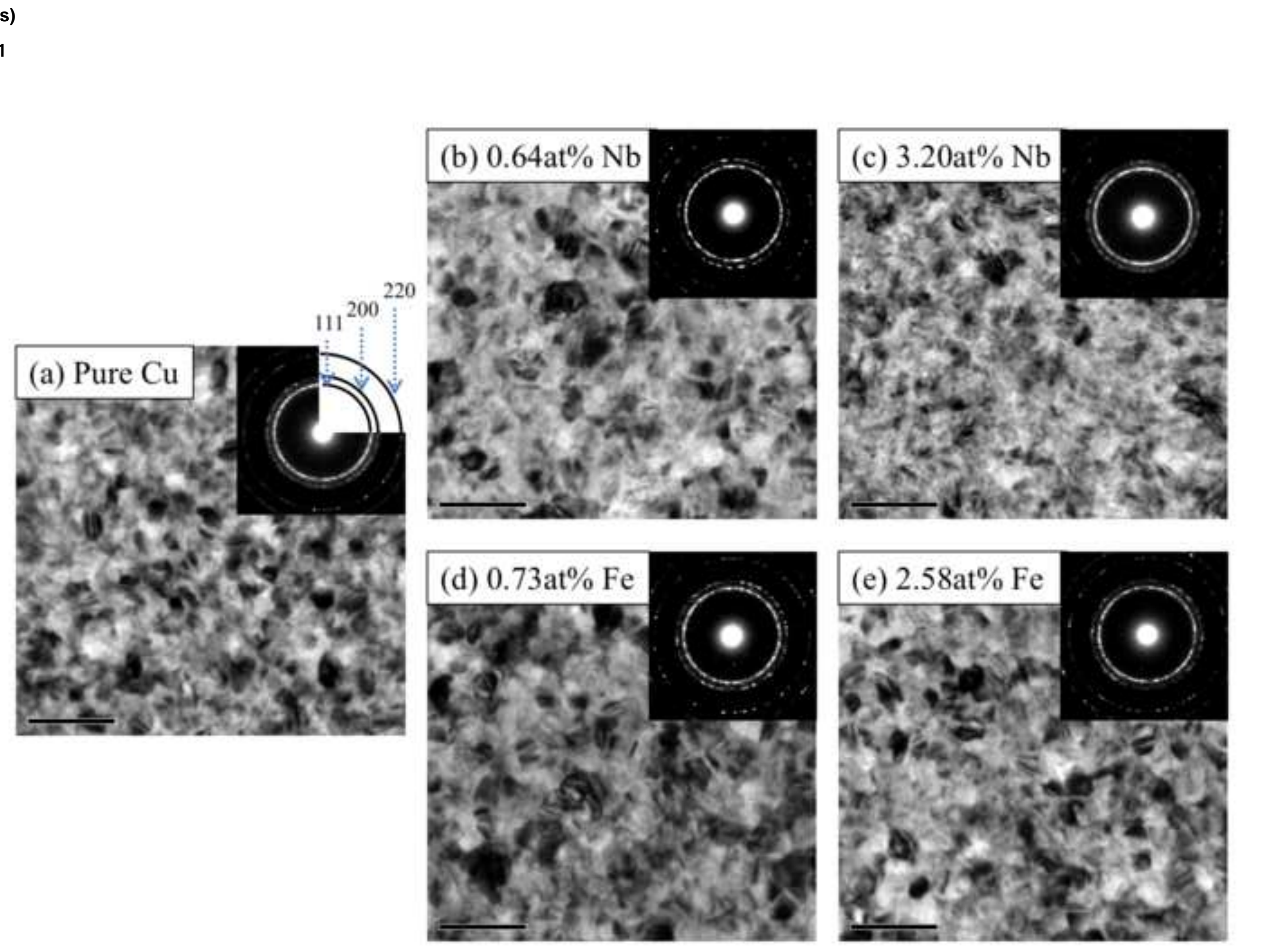

Figure 1

(b) $0.64 \mathrm{at} \% \mathrm{Nb}$

\author{
.

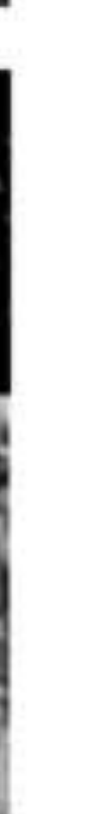


(a)

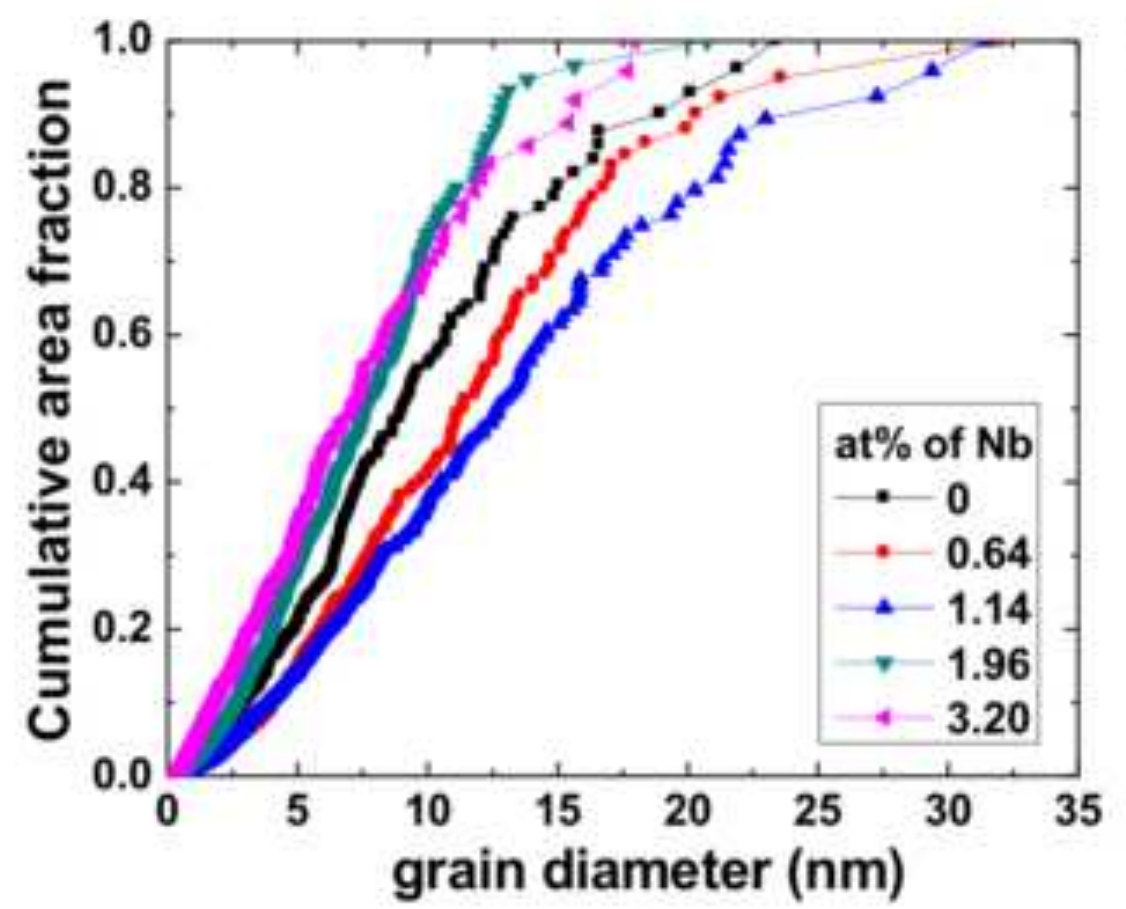

(c)

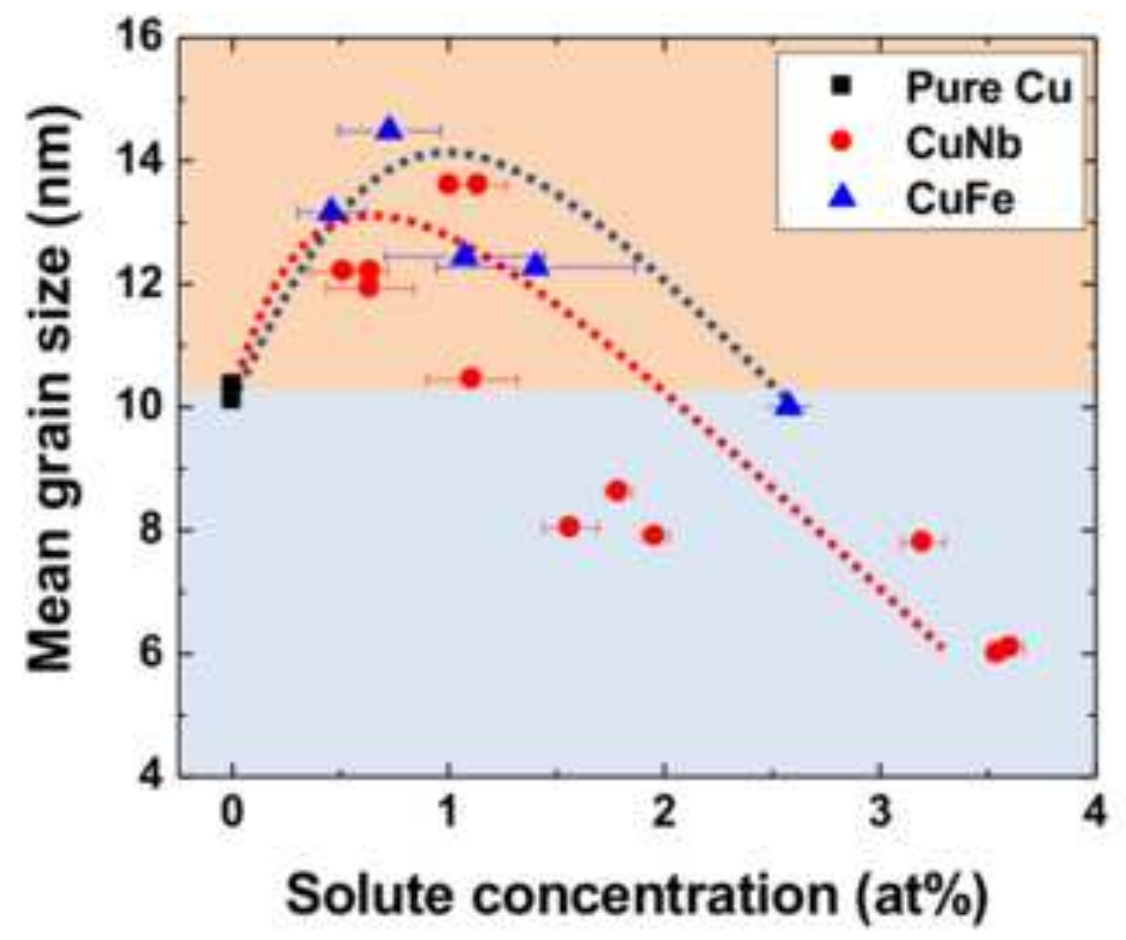

(b)

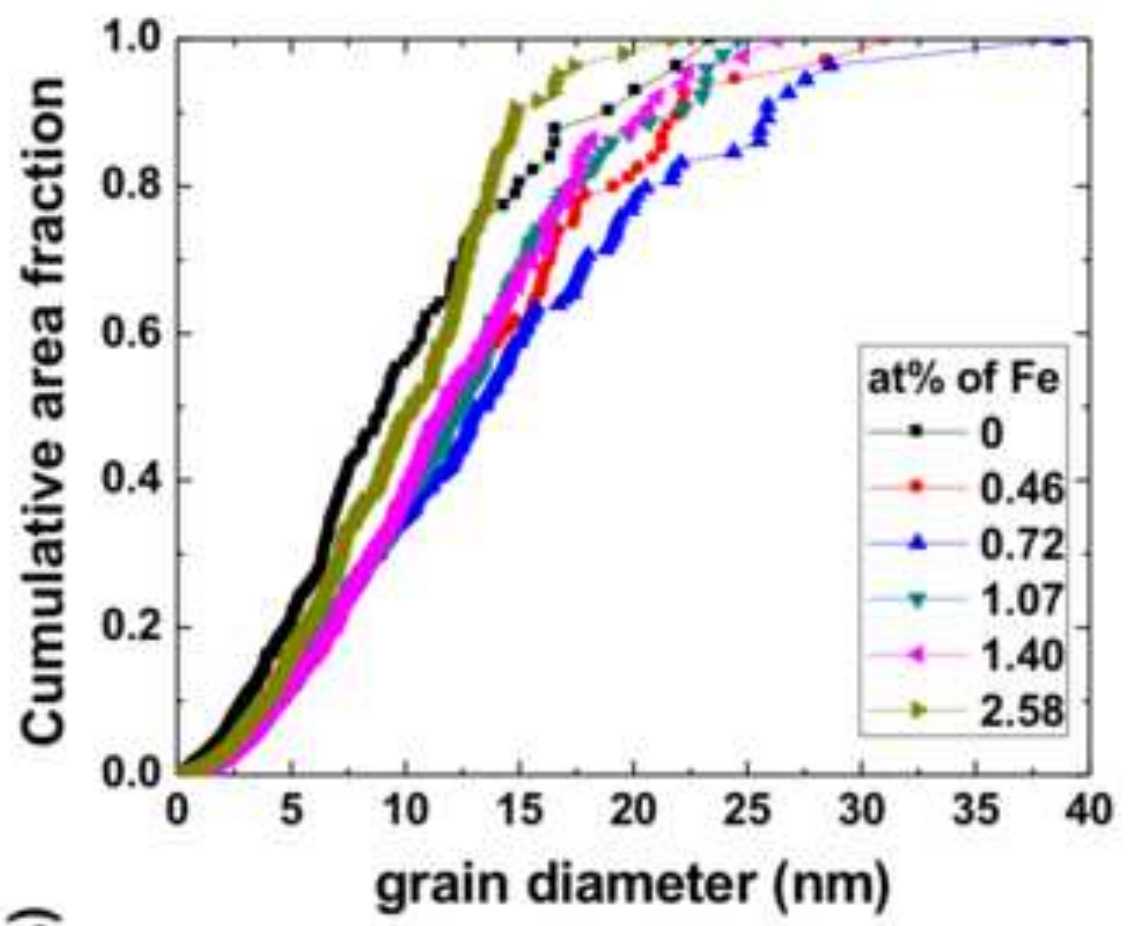

(d)

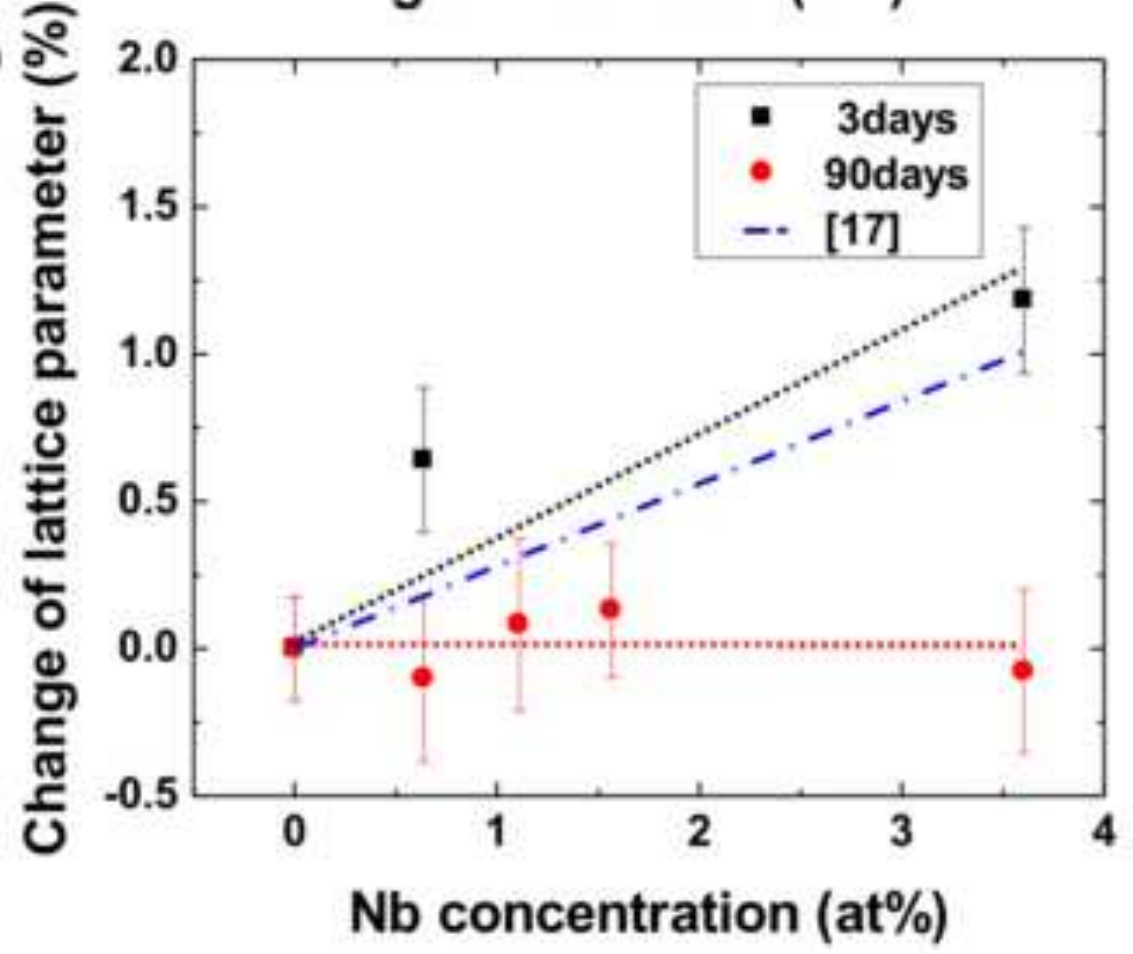




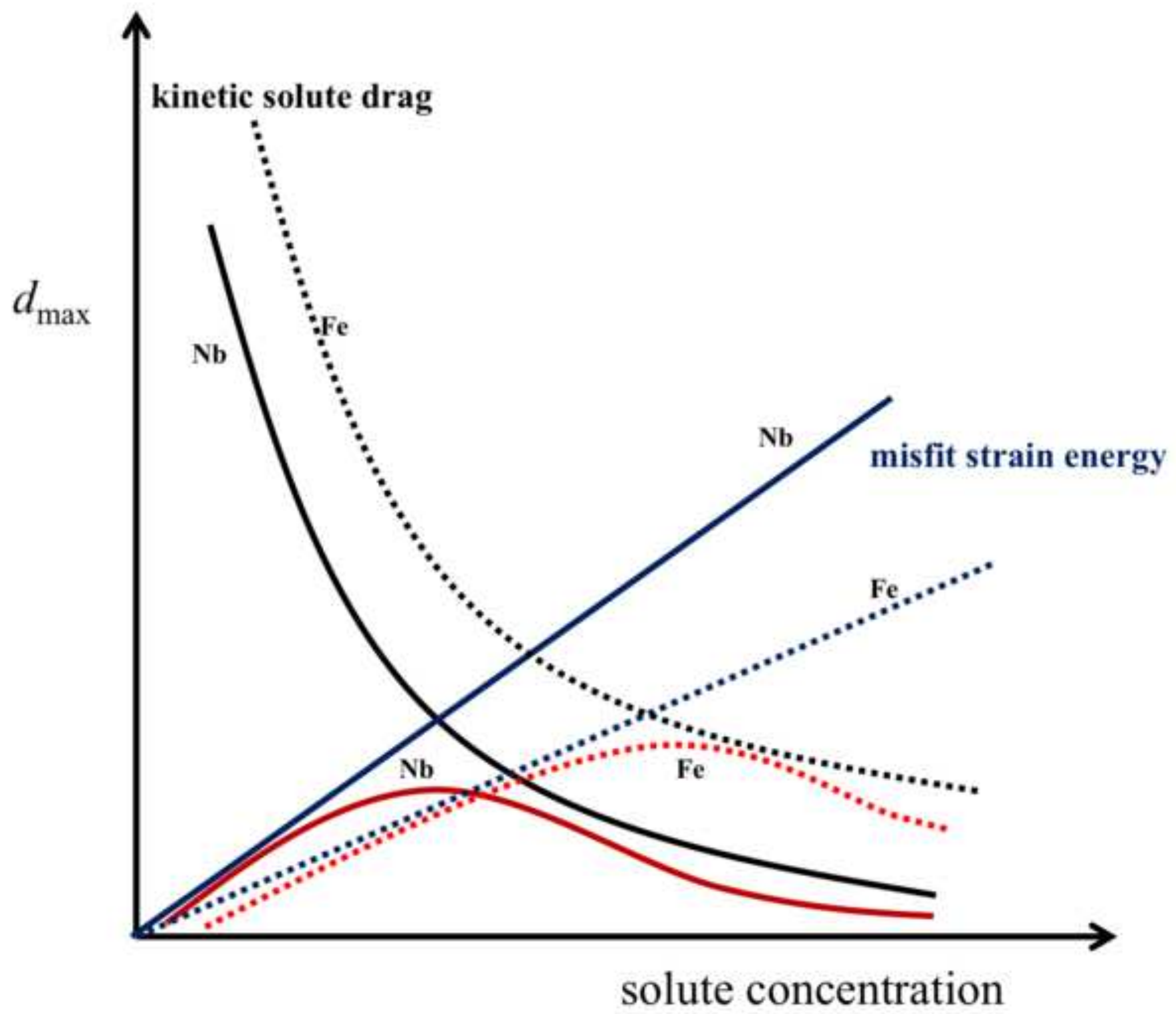




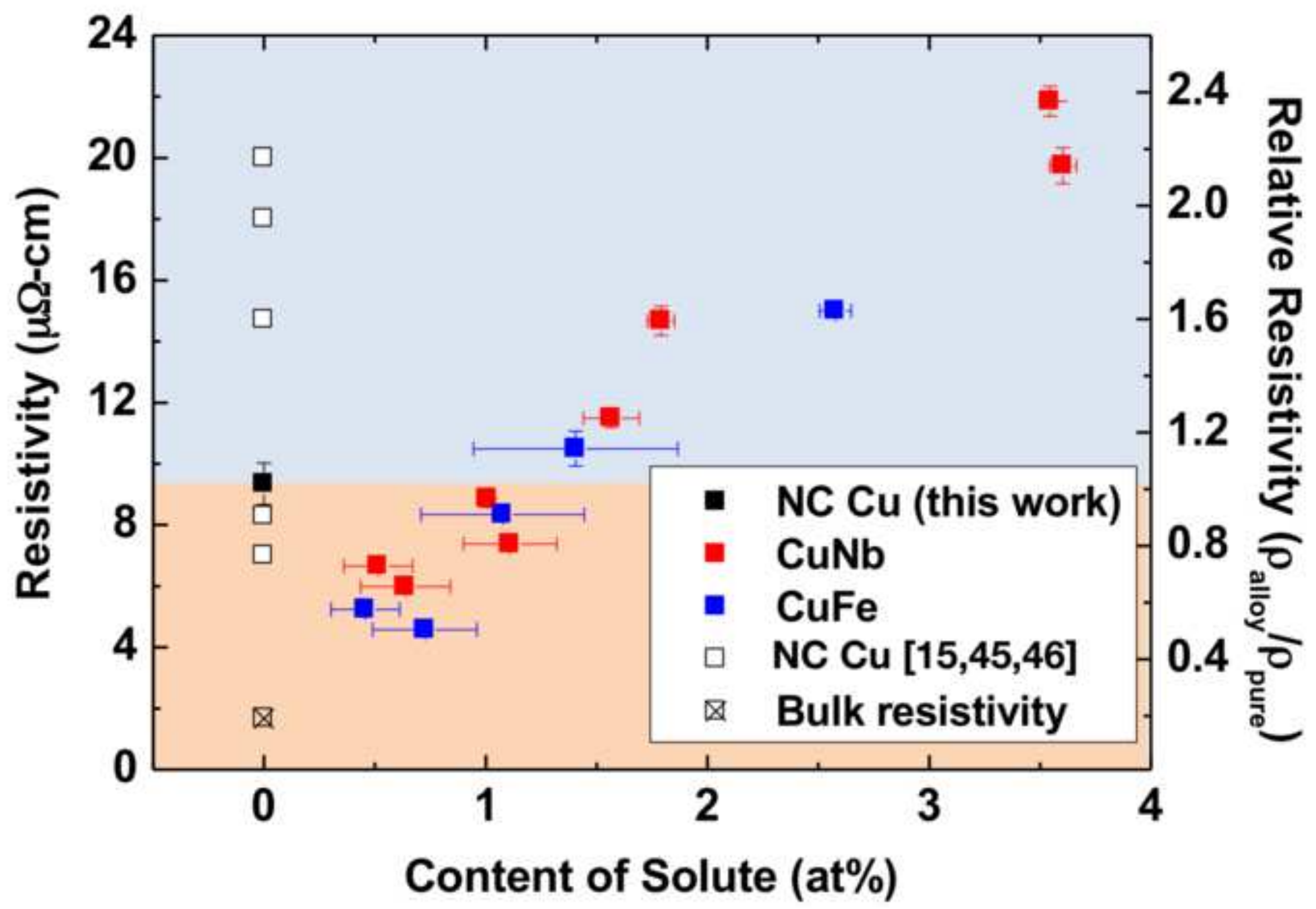




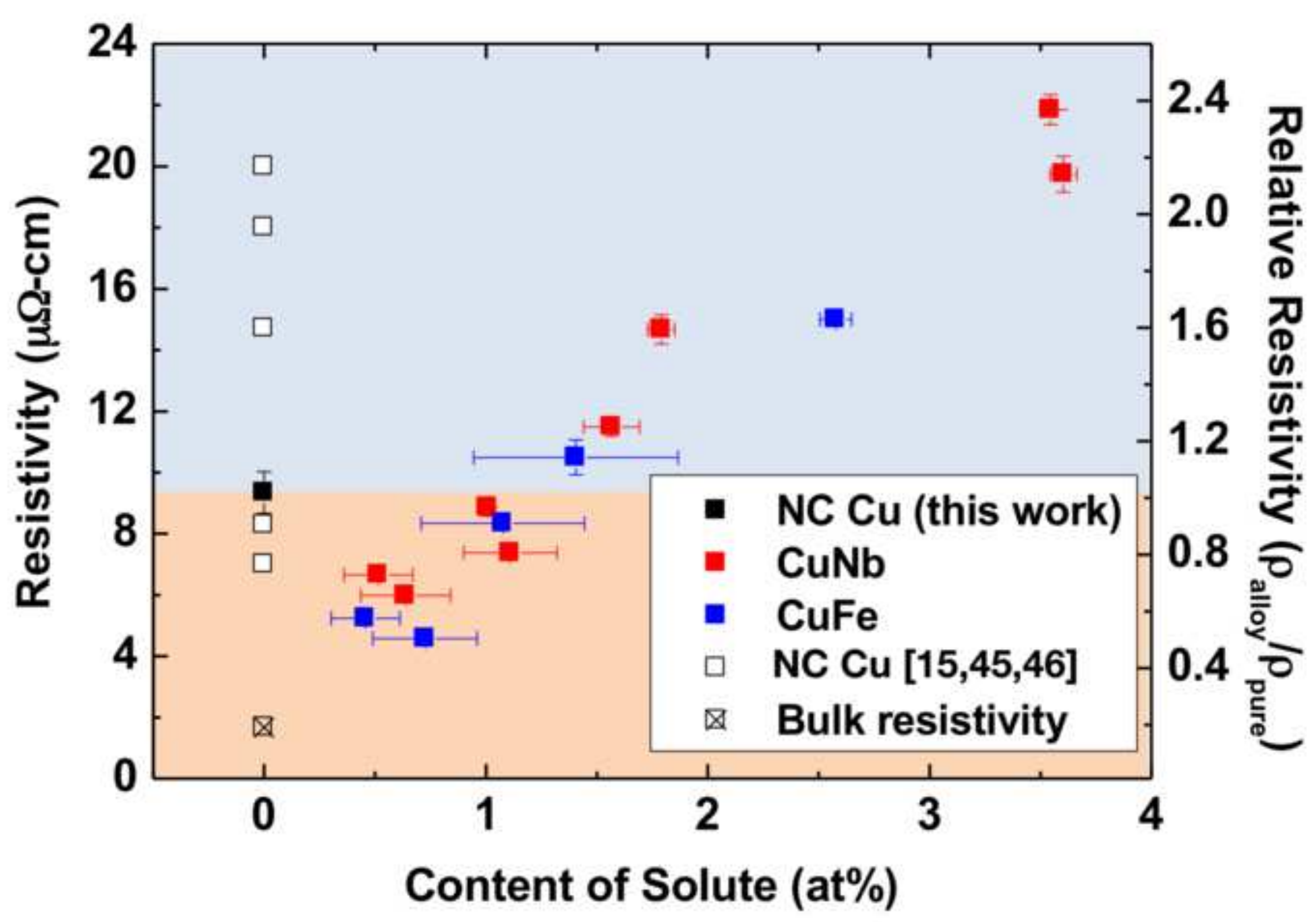

